\title{
Riesz inequality for the system of root functions of second order ordinary differential operator
}

\author{
AYGUN GARAYEVA \\ Department of Computational Mathematics \\ Baku State University \\ Z.Khalilov 23 \\ Baku, AZERBAIJAN
}

\begin{abstract}
An ordinary differential operator of second order with coefficients is considered. The Riesz property of the system of root functions of the given operator is studied. The criterion of Bessel property in $L_{Q}$ f root functions system is established and use it to obtain sufficient conditions for the Riesz property of a system of normalized root functions of this operator in $L_{p}$
\end{abstract}

Key-Words: - Root functions, Bessel property criterion, Riesz property

Received: November 23, 2019. Revised: April 4, 2020. Accepted: April 16, 2020. Published: April 27, 2020.

\section{Introduction}

We consider the differential operator

$$
L u=u^{\prime \prime}+P_{1}(x) u^{\prime}+P_{2}(x) u,
$$

in the interval $G=(0,1)$ with summable coefficients $P_{l}(x) \in L_{1}(G), l=1,2$.

The root functions of the operator $L$ are understood in generalized interpretation (without respect to boundary conditions) [1] .

Consider an arbitrary system $\left\{u_{k}(x)\right\}_{k=1}^{\infty}$ consisting of eigen-function and associated functions (root functions) of the operator $L$. Let $\left\{\lambda_{k}\right\}_{k=1}^{\infty}$ be the corresponding system of eigenvalues of this operator. We require that, together with each associated function of order $S, S \geq 1$, the system $\left\{u_{k}(x)\right\}_{k=1}^{\infty}$ also contains the corresponding eigenfunction and all associated functions 0 order $<S$. This means that each element of the system $\left\{u_{k}(x)\right\}_{k=1}^{\infty}$ is identically non-zero, is absolutely continuous together with its first order derivative on $\bar{G}$, and almost everywhere in $G$ satisfies the equation $L u_{k}+\lambda_{k} u_{k}=\theta_{k} u_{k-1}$, where $\theta_{k}$ equals either 0 (in this case $u_{k}(x)$ is an eigenfunction), or 1 (in this case $u_{k}(x)$ is an associated function of order $r \geq 1$, and $u_{k-1}(x)$ is an associated function of order $r-1$,

$$
\begin{gathered}
\lambda_{k}=\lambda_{k-1}=\ldots=\lambda_{k-r}, \\
\theta_{k}=\theta_{k-1}=\ldots=\theta_{k-r+1}=1
\end{gathered}
$$

and $\theta_{k-r}=0$ ).

The highest order of root functions responding to the given eigen function will be called the rank of this eigen function.

In such a generalized understanding of root functions V.A. Il'in [1] first established the Bessel property, necessary and sufficient conditions of unconditional basicity in $L_{2}$ of the system of root functions of the operator $L$ for $P_{1}(x) \equiv 0$, $P_{2}(x) \in L_{1}(G)$.

In the papers [2-9] these and other problems were studied for a higher order differential operator. This time, for the Bessel property it was always assumed that $P_{1}(x) \in L_{2}(G)$.

In the present paper we study the Bessel and Riesz property for the system of root functions of the operator $L$ with the coefficients $P_{l}(x) \in L_{1}(G)$, $l=1,2$

Definition 1. The system $\left\{v_{k}(x)\right\}_{k=1}^{\infty} \subset L_{2}(G)$ is called Bessel (or satisfies the Bessel inequality) if 
there exists a constant $M$ such that for any function $f(x) \in L_{2}(G)$ the following inequality is fulfilled:

$$
\left(\sum_{k=1}^{\infty} \|\left(f, v_{k}\right)^{2}\right)^{1 / 2} \leq M\|f\|_{2} \text {. }
$$

Definition 2. The system $\left\{v_{k}(x)\right\}_{k=1}^{\infty} \subset L_{q}(G)$ satisfies the Riesz inequiality in there exists a constant $M=M(P)$ such that

$$
\left(\sum_{k=1}^{\infty} \|\left(f, v_{k}\right)^{q}\right)^{1 / q} \leq M\|f\|_{p}, q=p /(p-1),
$$

for an arbitrary function

$$
f(x) \in L_{p}(G), 1<p \leq 2 .
$$

In the paper we prove the following assertions.

Theorem 1 (Sufficient conditions for the Riesz property). Let $P(x) \in L_{1}(G), l=1,2$ and there exist such a constant $C_{0}$ that

$$
\left|\operatorname{Im} \mu_{k}\right| \leq C_{0}, k=1,2, \ldots .
$$

Then for the Riesz property of the system $\left\{u_{k}(x)\left\|u_{k}\right\|_{q}^{-1}\right\}_{k=1}^{\infty}$ it is sufficient the existence of a constant $M_{1}$ such that

$$
\sum_{\tau \leq \operatorname{Re} \mu_{k} \leq \tau+1} \leq M_{1},
$$

where $\tau$ is an arbitrary non-negative number, $\mu_{k}=\sqrt{\lambda_{k}}, \operatorname{Re} \mu_{k} \geq 0$.

Theorem 2 (Bessel property criterion). Let $P_{l}(x) \in L_{1}(G), \quad l=1,2$; the rank of eigen functions be uniformly bounded and condition (1) is satisfied.

Then for the Bessel property of the system $\left\{u_{k}(x)\left\|u_{k}\right\|_{2}^{-1}\right\}_{k=1}^{\infty}$ in $L_{2}(G)$ it is necessary and sufficient the existence of a constant $M_{1}$ such that condition (2) is satisfied.

Remark. In a sufficient part of the Theorem 2, the condition of uniform boundedness of the rank of eigen functions is knowingly fulfilled, because it is a corollary of inequality (2).

Firstly we prove the Theorem 2 . Necessity. We fix an arbitrary number $\tau \geq 0$. Let

$$
\begin{gathered}
Q_{\tau}=\left\{k: \tau \leq \operatorname{Re} \mu_{k} \leq \tau+1,\left|\operatorname{Im} \mu_{k}\right| \leq C_{0}\right\}, \\
R_{0}=\left(n_{0}\left(1+C_{0}\right)^{3 / 2}\right)^{-1},
\end{gathered}
$$

where $n_{0} \geq 1$ is chosen so that $R_{0} \leq 1 / 4$ and for any set $E \subset \bar{G}$, mes $E \leq 2 R_{0}$, the following inequality is fulfilled:

$$
\omega\left(R_{0}\right)=\sup _{E \in \bar{G}}\left\{\left\|P_{l}\right\|_{1, E}, l=1,2\right\} \leq N_{0}^{-1},
$$

where $\left\|P_{l}\right\|_{1, E}=\int_{E}\left|P_{l}(x)\right| d x,\left(\|\cdot\|_{1}=\|\cdot\|_{1, G}\right) ; N_{0}$ is a positive number whose choice of value will be determined later.

Let $x \in[0,1 / 2], k \in Q_{\tau}$. We write the mean value formula [1] for the points $x, x+t, x+2 t$ at $t \leq R_{0}$

$$
\begin{gathered}
u_{k}(x)=2 u_{k}(x+t) \cos \mu_{k} t-u_{k}(x-2 t)+ \\
+\frac{1}{\mu_{k}} \int_{x}^{x+2 t}\left\{P_{1}(\xi) u_{k}^{\prime}(\xi)+P_{2}(\xi) u_{k}(\xi)-\theta_{k} u_{k-1}(\xi)\right\} \times \\
\times \sin \mu_{k}(|x+t-\xi|-t) d \xi
\end{gathered}
$$

Since by the theorem conditions the rank of eigenfunctions is uniformly bounded, then it suffices to consider only the eigen-functions $u_{k}(x)$, i.e. the case $\theta_{k}=0$. Then adding and subtracting in the right hand of formula the expression (4) $2 u_{k}(x+t) \cos \pi t$ and by using the identity $\cos \alpha-\cos \beta=2 \sin ((\alpha+\beta) / 2) \sin ((\beta-\alpha) / 2)$ and applying the operation $R_{0}^{-1} \int_{0}^{R_{0}} d t$, we obtain

$$
\begin{gathered}
u_{k}(x)=R_{0}^{-1}\left(u_{k}, v\right)+4 R_{0}^{-1} \int_{0}^{R_{0}} u_{k}(x+t) \\
\sin \frac{\mu_{k}+\tau}{2} t \sin \frac{\tau-\mu_{k}}{2} t d t+ \\
+\left(R_{0} \mu_{k}\right)^{-1} \int_{0}^{R_{0}} \int_{x}^{x+2 t}\left\{P_{1}(\xi) u_{k}^{\prime}(\xi)+\right. \\
\left.+P_{2}(\xi) u_{k}(\xi)\right\} \sin \mu_{k}(|x+t-\xi|-t) d \xi d t,
\end{gathered}
$$

where $v(t)=2 \cos \tau(x-t)-\frac{1}{2}$ for $t \in\left[x, x+R_{0}\right]$, $v(t)=-1 / 2$ for $t \in\left[x+R_{0}, x+2 R_{0}\right]$ and $v(t)=0$ for $t \notin\left[x, x+2 R_{0}\right]$. Taking into account the shift formula (see [1]) 


$$
\begin{gathered}
u_{k}(x \pm t)=u_{k}(x) \cos \mu_{k} t \pm \\
\pm \mu_{k}^{-1} \sin \mu_{k} t u_{k}^{\prime}(x) \pm \\
\pm \mu_{k}^{-1} \int_{x}^{x \pm t}\left\{P_{1}(\xi) u_{k}^{\prime}(\xi)+\right. \\
\left.+P_{2}(\xi) u_{k}(\xi)-\theta_{k} u_{k-1}(\xi)\right\} \times \\
\times \sin \mu_{k}(|x-\xi|-t) d \xi
\end{gathered}
$$

for $\theta_{k}=0$ in the second addend from the right hand side of relation (5), we get

$$
\begin{gathered}
u_{k}(x)=R_{0}^{-1}\left(u_{k}, v\right)+4 R_{0}^{-1} \times \\
\times \int_{0}^{R_{0}} \cos \mu_{k} t \sin \frac{\mu_{k}+\tau}{2} t \sin \frac{\tau-\mu_{k}}{2} t d t+ \\
+4\left(\mu_{k} R_{0}\right)^{-1} u_{k}^{\prime}(x) \int_{0}^{R_{0}} \sin \mu_{k} t \sin \frac{\mu_{k}+\tau}{2} t \times \\
\times \sin \frac{\tau-\mu_{k}}{2} t d t+4\left(\mu_{k} R_{0}\right)^{-1} \times \\
\times \int_{0}^{R_{0}} \sin \frac{\mu_{k}+\tau}{2} t \sin \frac{\tau-\mu_{k}}{2} d t \times \\
\times \int_{x}^{x+t}\left\{P_{1}(\xi) u_{k}^{\prime}(\xi)+P_{2}(\xi) u_{k}(\xi)\right\} \times \\
\times \sin \mu_{k}(|x-\xi|-t) d \xi d t+ \\
+\left(\mu_{k} R_{0}\right)^{-1} \int_{0}^{R_{0} x+2 t}\left\{P_{1}^{x}(\xi) u_{k}^{\prime}(\xi)+\right. \\
\left.+P_{2}(\xi) u_{k}(\varsigma)\right\} \sin \mu_{k}(|x+t-\xi|-t) d \xi d t= \\
=R_{0}^{-1}\left(u_{k}, v\right)+\sum_{j=1}^{4} \mathrm{~T}_{j} .
\end{gathered}
$$

Let as estimate the integrals $T_{j,} j=\overline{1,4}$.

Taking into account $k \in Q_{\tau}$ and using the inequality

$$
|\sin z| \leq 2,|\cos z| \leq 2,|\sin z| \leq 2|z|,
$$

for $\operatorname{Im} z \leq 1$ we get

$$
\begin{aligned}
\left|T_{1}\right| \leq & 8 R_{0}\left|\tau-\mu_{k}\right|\left|u_{k}(x)\right| \leq 8 R_{0}\left(1+C_{0}\right) \times \\
& \times\left|u_{k}(x)\right| \leq 8 n_{0}^{-1}\left|u_{k}(x)\right| \\
\left|T_{2}\right| \leq & 8 R_{0}\left|\tau-\mu_{k}\right|\left|\mu_{k}^{-1}\right|\left|u_{k}^{\prime}(x)\right| \leq 8 R_{0}\left(1+C_{0}\right) \times \\
& \times\left|\mu_{k}^{-1}\right|\left|u_{k}^{\prime}(x)\right| \leq 8 n_{0}^{-1}\left|\mu_{k}\right|^{-1}\left|u_{k}^{\prime}(x)\right|
\end{aligned}
$$

We estimate $T_{3}$ for $\left|\mu_{k}\right| \geq 1, k \in Q_{\tau}$. Using the estimations (see [6])

$$
\begin{gathered}
\left\|u_{k}^{(s)}\right\|_{\infty} \leq C_{1}\left(1+\left|\mu_{k}\right|\right)^{s}\left(1+\left|\operatorname{Im} \mu_{k}\right|\right)^{1 / p} \times \\
\times\left\{\left|u_{k}\left\|_{p}+\theta_{k}\left|\mu_{k}\right|^{-1}\left(1+\left|\operatorname{Im} \mu_{k}\right|\right)^{-1}|| u_{k-1}\right\|_{p}\right\},\right. \\
1 \leq p<\infty, s=0,1 ; \\
\left\|u_{k}^{\prime}\right\|_{p} \leq C_{2}\left(1+\left|\mu_{k}\right|\right)\left\{\left.\left|u_{k} \|_{p}+\theta_{k}\right| \mu_{k}\right|^{-1} \times\right. \\
\left.\times\left(1+\left|\operatorname{Im} \mu_{k}\right|\right)^{-1}\left\|u_{k-1}\right\|_{p}\right\}, \quad p \geq 1,
\end{gathered}
$$

for $\theta_{k}=0$ and applying the above elementary inequalities, we have

$$
\begin{gathered}
\left|T_{3}\right| \leq 32 R_{0}\left|\mu_{k}^{-1}\right| \int_{0}^{R_{0} x+t} \int_{x}\left|P_{1}(\xi)\right|\left|u_{k}^{\prime}(\xi)\right| d \xi d t+ \\
+32 R_{0} \int_{0}^{R_{0} x+t} \int_{x}\left|P_{2}(\xi)\right| d \xi d t \leq 32 \omega\left(R_{0}\right)\left|\mu_{k}\right|^{-1}\left\|u_{k}^{\prime}\right\|_{\infty}+ \\
+16 R_{0} \omega\left(R_{0}\right) \mid u_{k} \|_{\infty} \leq 32 \omega\left(R_{0}\right) \times \\
C_{1}\left(1+\left|\mu_{k}\right|^{-1}\right)\left(1+\left|\operatorname{Im} \mu_{k}\right|\right)^{1 / 2}\left\|u_{k}\right\|_{2}+ \\
+16 R_{0} \omega\left(R_{0}\right) C_{1}\left(1+\left|\operatorname{Im} \mu_{k}\right|\right)^{1 / 2}\left\|u_{k}\right\|_{2} \leq \\
\leq 64 \omega\left(R_{0}\right)\left(1+C_{0}\right)^{1 / 2} C_{1}\left\|u_{k}\right\|_{2}+ \\
+16 C_{1}\left(1+C_{0}\right)^{1 / 2} R_{0} \omega\left(R_{0}\right)\left\|u_{k}\right\|_{2} \leq \\
\leq C_{3} \omega\left(R_{0}\right)\left\|u_{k}\right\|_{2} \leq C_{4} N_{0}^{-1}\left\|u_{k}\right\|_{2} .
\end{gathered}
$$

The same estimation is fulfilled also for the integral $T_{4}$ for $k \in Q_{\tau},\left|\mu_{k}\right| \geq 1$. Consequently, from (7) we get

$$
\begin{gathered}
\left|u _ { k } ( x ) \left\|\left|u_{k}\left\|_{2}^{-1} \leq R_{0}^{-1}\left|\left(u_{k}, v\right)\right|+8 n_{0}^{-1} \mid u_{k}(x)\right\|\left\|_{k}\right\|_{2}^{-1}+\right.\right.\right. \\
+8 n_{0}^{-1}\left|\mu_{k}\right|^{-1} \mid u_{k}^{\prime}(x)\left\|u_{k}\right\|_{2}^{-1}+C_{4} N_{0}^{-1}
\end{gathered} .
$$

By virtue of symmetry (see formulas (4), (6)) this inequality is valid in the case $x \in[1 / 2,1]$ as well. This time the function $v(t)$ is determined by the formula: $v(t)=-1 / 2$ for $t \in\left[x-2 R_{0}, x-R_{0}\right]$, $v(t)=2 \cos \tau(x-t)-1 / 2 \quad$ for $t \in\left[x-R_{0}, x\right]$, $v(t)=0$ for $t \notin\left[x-2 R_{0}, x\right]$.

Hence, for $n_{0} \geq 16$ it follows that

$$
\begin{gathered}
\left.\left|u_{k}(x) \|\right| u_{k}\right|_{2} ^{-1} \leq 2 R_{0}^{-1}\left|\left(u_{k}, v\right)\right|+16 n_{0}^{-1}\left|\mu_{k}\right|^{-1} \times \\
\times\left|u_{k}^{\prime}(x)\|\mid\| u_{k} \|_{2}^{-1}+2 C_{4} N_{0}^{-1}\right.
\end{gathered}
$$

We square every part of this inequality, integrate with respect to $x$ from 0 to 1 , sum over $k \in Q_{\tau}^{\prime}=\left\{k: k \in Q_{\tau},\left|\mu_{k}\right| \geq 1\right\}$, and by using estimate 
(9) for $p=2, \theta_{k}=0$ apply the Bessel inequality, and with regard to the equality $\|v\|_{2}^{2}=O\left(R_{0}\right)$, get

$$
\sum_{k \in Q_{\tau}^{\prime}} 1 \leq O\left(R_{0}^{-1}\right)+\left\{C_{7} n_{0}^{-2}+C_{8} N_{0}^{-2}\right\} \sum_{k \in Q_{\tau}^{\prime}} 1 .
$$

Choosing the numbers $n_{0}$ and $N_{0}$ so that $C_{7} n_{0}^{-2}+C_{8} N_{0}^{-2} \leq \frac{1}{2}$, we arrive at the inequality $\sum_{k \in Q_{\tau}^{\prime}} 1 \leq$ const. Consequently, for $\left|\mu_{k}\right| \geq 1$ the necessity of condition (2) is established.

For $\left|\mu_{k}\right|<1$ the validity of condition (2) is proved in the following way. We consider the equation $L u_{k}-2 u_{k}+\lambda_{k}^{\prime} u_{k}=0$, where $\lambda_{k}^{\prime}=\lambda_{k}+2,\left|\lambda_{k}\right|<1$. Then $\left|\operatorname{Re} \lambda_{k}^{\prime}\right| \geq 1$ and the system $\left\{u_{k}\right\}$ does not change. Therefore inequality (2) is fulfilled in the case $\left|\mu_{k}\right|<1$ as well. The necessity of condition (2) is established.

Sufficiency. Let conditions (1) and (2) be fulfilled. Prove that the system $\left\{u_{k}(x)\left\|u_{k}\right\|_{2}^{-1}\right\}_{k=1}^{\infty}$ is Bessel in $L_{2}(G)$. By formula (6), conditions (1) and (2) for the convergence of the series $\sum_{k=1}^{\infty}\left|\left(f, u_{k}\left\|u_{k}\right\|_{2}^{-1}\right)\right|^{2}$ it suffices to prove the validity of the following inequalities:

$$
\begin{aligned}
& \left.\left.\sum_{\left|\mu_{k}\right| \geq 1}\right|_{0} ^{1} \overline{f(t)} \cos \mu_{k} t d t\right|^{2}\left\|u_{k}\right\|_{2}^{-2}\left|u_{k}(0)\right|^{2} \leq C\|f\|_{2}^{2} ; \\
& \left.\left.\sum_{\left|\mu_{k}\right| \geq 1}\left|\mu_{k}\right|^{-2}\right|_{0} ^{1} \overline{f(t)} \sin \mu_{k} t d t\right|^{2}\left|u_{k}^{\prime}(0)\right|^{2}\left\|u_{k}\right\|_{2}^{-2} \leq C\|f\|_{2}^{2} \text {; } \\
& \sum_{\left|\mu_{k}\right| \geq 1}\left|\mu_{k}\right|^{-2} \mid \int_{0}^{1} \overline{f(t)} \int_{0}^{t} P_{1}(\xi) u_{k}^{\prime}(\xi) \times \text {; } \\
& \times\left.\sin \mu_{k}(\xi-t) d \xi d t\right|^{2}\left\|u_{k}\right\|_{2}^{-2} \leq C\|f\|_{2}^{2} \\
& \left.\sum_{\left|\mu_{k}\right| \geq 1}\left|\mu_{k}\right|^{-2} \int_{0}^{1} \overline{f(t)}\right|_{0} ^{t} P_{2}(\xi) u_{k}(\xi) \times \text {; } \\
& \times\left.\sin \mu_{k}(\xi-t) d \xi d t\right|^{2}\left\|u_{k}\right\|_{2}^{-2} \leq C\|f\|_{2}^{2} \\
& \sum_{\left|\mu_{k}\right| \geq 1}\left|\theta_{k} \int_{0}^{1} \overline{f(t)} \int_{0}^{t} u_{k-1}(\xi) \sin \mu_{k}(\xi-t) d \xi d t\right|^{2} \times \\
& \times\left\|u_{k}\right\|_{2}^{-2}\left|\mu_{k}\right|^{-2} \leq C\|f\|_{2}^{2}
\end{aligned}
$$

where $f \in L_{2}(G)$.
The validity of these inequalities except the third inequality, was established in [1]. Prove the validity of the third inequality. Denote

$$
\eta(t, \xi)= \begin{cases}f(t+\xi), & 0 \leq t \leq 1-\xi \\ 0, & 1-\xi<t \leq 1,\end{cases}
$$

where $\xi \in[0,1]$.

Then

$$
\begin{aligned}
& S_{k}=\left|\mu_{k}\right|^{-2} \times \\
& \times\left|\int_{0}^{1} \overline{f(t)} \int_{0}^{t} P_{1}(\xi) u_{k}^{\prime}(\xi) \sin \mu_{k}(\xi-t) d \xi d t\right|^{2}\left\|u_{k}\right\|_{2}^{-2}= \\
& =\int_{0}^{t} P_{1}(\xi) u_{k}^{\prime}(\xi) \mu_{k}^{-1}\left\|u_{k}\right\|_{2}^{-1} \int_{0}^{1} \overline{\eta(t, \xi)} \sin \mu_{k} t d t d \xi \times
\end{aligned}
$$

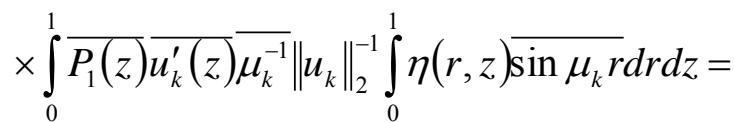

$$
\begin{aligned}
& =\int_{0}^{1} \int_{0}^{1} P_{1}(\xi) \overline{P_{1}(z)} u_{k}^{\prime}(\xi) \mu_{k}^{-1}\left\|u_{k}\right\|_{2}^{-1} \overline{u^{\prime}(z)} \overline{\mu_{k}^{-1}}\left\|u_{k}\right\|_{2}^{-1} \times \\
& \times \int_{0}^{1} \overline{\eta(t, \xi)} \sin \mu_{k} t d t \int_{0}^{1} \eta(r, z) \overline{\sin \mu_{k} r} d r d \xi d z .
\end{aligned}
$$

Applying here the estimation

$$
\left\|u_{k}^{\prime}\right\|_{\infty} \leq C_{9}\left(1+\left|\mu_{k}\right|\right)\left\|u_{k}\right\|_{2}
$$

(see $[10]$ ), we get that at $\left|\mu_{k}\right| \geq 1$ for $S_{k}$ the following inequality is fulfilled:

$$
\begin{aligned}
& S_{k} \leq C_{10} \int_{0}^{1} \int_{0}^{1}\left|P_{1}(\xi)\right|\left|P_{1}(z)\right| \int_{0}^{1} \overline{\eta(t, \xi)} \sin \mu_{k} t d t \mid \times \\
& \times\left|\int_{0}^{1} \overline{\eta(r, z)} \sin \mu_{k} r d r\right| d \xi d z .
\end{aligned}
$$

For an arbitrary finite subset $J^{\prime}$ of the set of indices $J=\left\{k:\left|\mu_{k}\right| \geq 1\right\}$ we obtain

$$
\begin{gathered}
\sum_{k \in J^{\prime}} S_{k} \leq\left. C_{10} \sum_{k \in J^{\prime}} \int_{0}^{1} \int_{0}^{1}\left|P_{1}(\xi)\right|\left|P_{1}(z)\right|\right|_{0} ^{1} \overline{\eta(t, \xi)} \sin \mu_{k} t d t \mid \times \\
\times\left|\int_{0}^{1} \overline{\eta(r, z)} \sin \mu_{k} r d r\right| d \xi d z= \\
=C_{10} \int_{0}^{1} \int_{0}^{1}\left|P_{1}(\xi)\right|\left|P_{1}(z)\right| \times
\end{gathered}
$$




$$
\begin{aligned}
& \times \sum_{k=J^{\prime}}\left|\int_{0}^{1} \overline{\eta(t, \xi)} \sin \mu_{k} t d t\right| \| \int_{0}^{1} \overline{\eta(r, z)} \sin \mu_{k} r d r \mid d \xi d z \leq \\
& \leq\left.\left. C_{10} \int_{0}^{1} \int_{0}^{1}\left|P_{1}(\xi)\right|\left|P_{1}(z)\right|\left|\sum_{k \in J^{\prime}}\right| \int_{0}^{1} \overline{\eta(t, \xi)} \sin \mu_{k} t d t\right|^{2}\right|^{1 / 2} \times \\
& \quad \times\left(\left.\sum_{k \in J^{\prime}}\left|\int_{0}^{1} \overline{\eta(r, z)} \sin \mu_{k} r d r\right|^{2}\right|^{1 / 2} d \xi d z \leq\right. \\
& \leq C_{11} \int_{0}^{1} \int_{0}^{1}\left|P_{1}(\xi) P_{1}(z)\|\eta(\cdot, \xi)\|\left\|_{2} \mid\right\| \eta(\cdot, z) \|_{2} d \xi d z .\right.
\end{aligned}
$$

Taking into account that for any $\xi \in[0,1]$ we have the inequality $\|\eta(\cdot, \xi)\|_{2} \leq\|f\|_{2}$, we obtain

$$
\sum_{k \in J^{\prime}} S_{k} \leq C_{11}\left\|P_{1}\right\|_{1}^{2}\|f\|_{2}^{2} \text {. }
$$

Hence, from arbitrariness of $J^{\prime} \subset J$, it follows the inequality $\sum_{k \in J} S_{k} \leq C_{12}\|f\|_{2}^{2}$.

The Theorem 2 is completely proved.

Proof of theorem 1. Let conditions (1) and (2) be fulfilled. Then by virtue of the sufficiency part of Theorem 2, for an arbitrary function $f(x) \in L_{2}(G)$ the following Bessel inequality is fulfilled:

$$
\left(\sum_{k=1}^{\infty}\left|\left(f, u_{k}\left\|u_{k}\right\|_{2}^{-1}\right)\right|^{2}\right)^{1 / 2} \leq M\|f\|_{2} .
$$

On the other hand, for any $f(x) \in L_{1}(G)$

$$
\sup _{k}\left|\left(f, u_{k} \mid\left\|u_{k}\right\|_{2}^{-1}\right)\right| \leq \sup _{k}\left\{\mid u_{k}\left\|_{\infty}\right\| u_{k} \|_{2}^{-1}\right\} \mid f \|_{1} .
$$

Hence, by virtue of the inequality

$$
\left\|u_{k}\right\|_{r} \leq c_{11}\left\|u_{k}\right\|_{\gamma}, \gamma \geq 1, r \geq 1(\sec [10])
$$

we get that for any function $f(x) \in L_{1}(G)$ the following inequality is valid:

$$
\sup _{k}\left\{\left|\left(f, u_{k}\left\|u_{k}\right\|_{2}^{-1}\right)\right|\right\} \leq M_{2}\|f\|_{2} .
$$

By virtue of the Riesz-Torin interpolational theorem (see [11], p. 144) from inequalities (11) and (13) it follows that the system $\left\{u_{k}(x)\left\|u_{k}\right\|_{2}^{-1}\right\}_{k=1}^{\infty}$ satisfies the Riesz inequality, i.e.

$$
\left(\sum_{k=1}^{\infty}\left|\left(f, u_{k}\left\|u_{k}\right\|_{2}^{-1}\right)\right|^{q}\right)^{1 / q} \leq M(p)\|f\|_{p}
$$

for $1<p \leq 2, q=p /(p-1)$.

Since

$$
\frac{u_{k}(x)}{\left\|u_{k}\right\|_{2}}=\frac{u_{k}(x)}{\left\|u_{k}\right\|_{q}} \frac{\left\|u_{k}\right\|_{q}}{\left\|u_{k}\right\|_{2}}
$$

and by virtue of inequality (12), the estimation

$$
\frac{\left\|u_{k}\right\|_{q}}{\left\|u_{k}\right\|_{2}} \leq C_{12}
$$

is fulfilled, then the system $\left\{u_{k}(x)\left\|u_{k}\right\|_{q}^{-1}\right\}_{k=1}^{\infty}$ satisfies the Riesz inequality as well, i.e. the inequality

$$
\begin{aligned}
& \left(\sum_{k=1}^{\infty} \mid\left(f, u_{k}\left\|u_{k}\right\|_{q}^{-1}\right)^{q}\right)^{1 / q} \leq \\
\leq & M_{2}(p)\|f\|_{p}, q=p /(p-1),
\end{aligned}
$$

is fulfilled for any $f(x) \in L_{p}(G), 1<p \leq 2$.

Theorem 2 is proved. In [12] and [13] some similar studies can be found.

\section{References:}

[1] Il'in V.A. On the unconditional basicity of systems of eigen and adjoint functions of second order differential operator on a closed interval Doklady AN SSSR 1983 Vol. 273 No.5 pp.10481053.

[2] Kerimov N.B. On the unconditional basicity of systems of eigen and adjoint functions of the fourth order differential operator Doklady AN SSSR 1986, Vol. 286 No.4 pp.803-808.

[3] Budaev V.D. The Bessel property and Riesz basicity of the system of root functions of differential operators 1,11 Differential Equations 1991, Vol.27 No.12 2033-2044 Vol.28 No.1 pp.2333.

[4] Lomov I.S. The Bessel inequality, Riesz theorem and unconditional basicity for root vectors of ordinary differential operators Vestnik Moskow 1992, No.5 pp. 42-52.

[5] Kurbanov V.M. On Hausedorff-Young inequality of root vector-functions of nth order differential operator Differential Equations 1997, Vol.33 No.3 pp.356367.

[6] Kurbanov V.M. On distribution of eigen values and Bessel criterion of root functions of differential operator 1,11 
Differential Equations, 2005, Vol.41 No.4 464-478 Vol.41 No.5 pp. 623-631

[7] Kurbanov V.M. A theorem on equivalent bases for a differential operator Doklady RAN, 2006, Vol.406 No.7 pp.17-20

[8] Kurbanov VM On analog of the Riesz theorem and basicity in $L_{p}$ of the system of root functions of differential operator 1 ,11 Differential Equations 2013, Vol.49 No.4 pp. 456-468.

[9] Kritskov L.V. Bessel property of the system of root functions of a secondorder singular operator on an interval Differential Equations 2018, Vol. 54 No.8 pp. 1032-1048.

[10] Kerimov N.R. Some properties of eigen and associate functions of ordinary differential operators Doklady AN SSSR, 1986, Vol.291 No.5 pp.1054-1055.

[11] Zigmund A. Trigonometric series. Cambridge, 1959, Translated under the title Trigonometricheskie ryady, Moscow 1965, vol.2

[12] Fatima Guliyeva, Relationship between an atomic decomposition of double and unary systems in grand-Lebesgue spaces, WSEAS Transactions on Mathematics, pp. 50-56, Volume 19, 2020

[13] Dzenan Gusic, Prime Geodesic Theorem for Compact Riemann Surfaces, International journal of circuits, systems and signal processing, NAUN, pp.747753, Volume 13, 2019 